\title{
The Effect of a Methoxy Substituent on the Vinylcyclobutane Carbon Migration
}

\author{
Celina C. Lasota, Phyllis A. Leber, ${ }^{*}$ Neil A. Strotman, ${ }^{\dagger}$ and Gloria S. Yen \\ Department of Chemistry, Franklin \& Marshall College, Lancaster, Pennsylvania 17604-3003
}

\section{Table of Contents}

Title page and detailed list of the contents of the Supporting Information (SI) ..............................S1

Figure S1: ${ }^{1} \mathrm{H}$ - and ${ }^{13} \mathrm{C}-\mathrm{NMR}$ Spectra of 8-exo-Methoxybicyclo[4.2.0]oct-2-en-7-one (3a) .............. S2

Figure S2: ${ }^{1} \mathrm{H}$ - and ${ }^{13} \mathrm{C}-\mathrm{NMR}$ Spectra of 8-endo-Methoxybicyclo[4.2.0]oct-2-en-7-one (3b)

contaminated with 8-exo-Methoxybicyclo[4.2.0]oct-2-en-7-one (3a) .............................S3

Figure S3: ${ }^{1} \mathrm{H}$ - and ${ }^{13} \mathrm{C}-\mathrm{NMR}$ Spectra of 8-exo-Methoxybicyclo[4.2.0] oct-2-ene (1a) ....................... S4

Figure S4: ${ }^{1} \mathrm{H}$ - and ${ }^{13} \mathrm{C}-\mathrm{NMR}$ Spectra of 8-endo-Methoxybicyclo[4.2.0]oct-2-ene (1b) ..................... S5

Figure S5: ${ }^{1} \mathrm{H}$ - and ${ }^{13} \mathrm{C}-\mathrm{NMR}$ Spectra of Bicyclo[2.2.2] oct-5-en-2-one (5) ..................................... S6

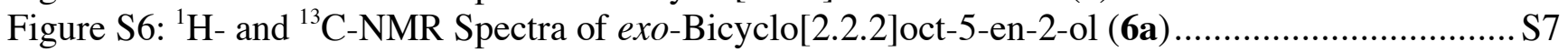

Figure S7: ${ }^{1} \mathrm{H}$ - and ${ }^{13} \mathrm{C}-\mathrm{NMR}$ Spectra of endo-Bicyclo[2.2.2] oct-5-en-2-ol (6b) contaminated

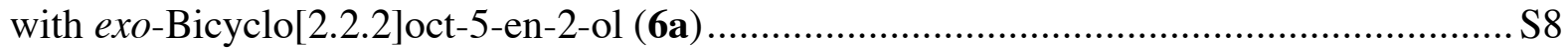

Figure S8: ${ }^{1} \mathrm{H}$ - and ${ }^{13} \mathrm{C}$-NMR Spectra of 5-exo-Methoxybicyclo[2.2.2]oct-2-ene (2a) ...................... S9

Figure S9: ${ }^{1} \mathrm{H}$ - and ${ }^{13} \mathrm{C}-\mathrm{NMR}$ Spectra of 5-endo-Methoxybicyclo[2.2.2]oct-2-ene (2b).................. S10

Figure S10: ${ }^{1} \mathrm{H}$ - and ${ }^{13} \mathrm{C}$-NMR Spectra of 7-exo-Methoxybicyclo[3.2.0]hept-2-ene (7a) ................. S11

Figure S11: ${ }^{1} \mathrm{H}$ - and ${ }^{13} \mathrm{C}$-NMR Spectra of 7-endo-Methoxybicyclo[3.2.0]hept-2-ene (7b) ............... S12

Figure S12: ${ }^{1} \mathrm{H}$ - and ${ }^{13} \mathrm{C}$-NMR Spectra of 5-exo-Methoxybicyclo[2.2.1]hept-2-ene (8a) ................. S13

Figure S13: ${ }^{1} \mathrm{H}$ - and ${ }^{13} \mathrm{C}-\mathrm{NMR}$ Spectra of 5-endo-Methoxybicyclo[2.2.1]hept-2-ene (8b) ............... S14

Table S1: Kinetic data for thermal reactions of $\mathbf{1 a}$ at $250{ }^{\circ} \mathrm{C}$ and

Figure S14: First-order Kinetic Rate Plot based on data in Table S1 ............................. S15

Table S2: Kinetic data for thermal reactions of $1 \mathbf{a}$ at $275^{\circ} \mathrm{C}$ and

Figure S15: First-order Kinetic Rate Plot based on data in Table S2 ............................S16

Table S3: Kinetic data for thermal reactions of $\mathbf{1 a}$ at $300{ }^{\circ} \mathrm{C}$ and

Figure S16: First-order Kinetic Rate Plot based on data in Table S3 ............................ S17

Table S4: Kinetic data for thermal reactions of $\mathbf{1 b}$ at $250{ }^{\circ} \mathrm{C}$ and

Figure S17: First-order Kinetic Rate Plot based on data in Table S4 ..............................S18

Table S5: Kinetic data for thermal reactions of $\mathbf{1 b}$ at $275^{\circ} \mathrm{C}$ and

Figure S18: First-order Kinetic Rate Plot based on data in Table S5 ............................... S19

Table S6: Kinetic data for thermal reactions of $\mathbf{1 b}$ at $300{ }^{\circ} \mathrm{C}$ and

Figure S19: First-order Kinetic Rate Plot based on data in Table S6............................. S20

Table S7: Temperature-dependent concentration data for thermal reaction of $\mathbf{1 a}$ and

Figure S20: Arrhenius plot for data on Table S7.

Table S8: Temperature-dependent concentration data for thermal reaction of $\mathbf{1 b}$ and

Figure S21: Arrhenius plot for data on Table S8

Figure S22: Sample GC chromatogram for thermal reactions of 1a and

Figure S23: Sample GC chromatogram for thermal reactions of $\mathbf{1 b}$ 
- $\mathrm{Et}_{2} \mathrm{O}$

$* \mathrm{H}_{2} \mathrm{O}$
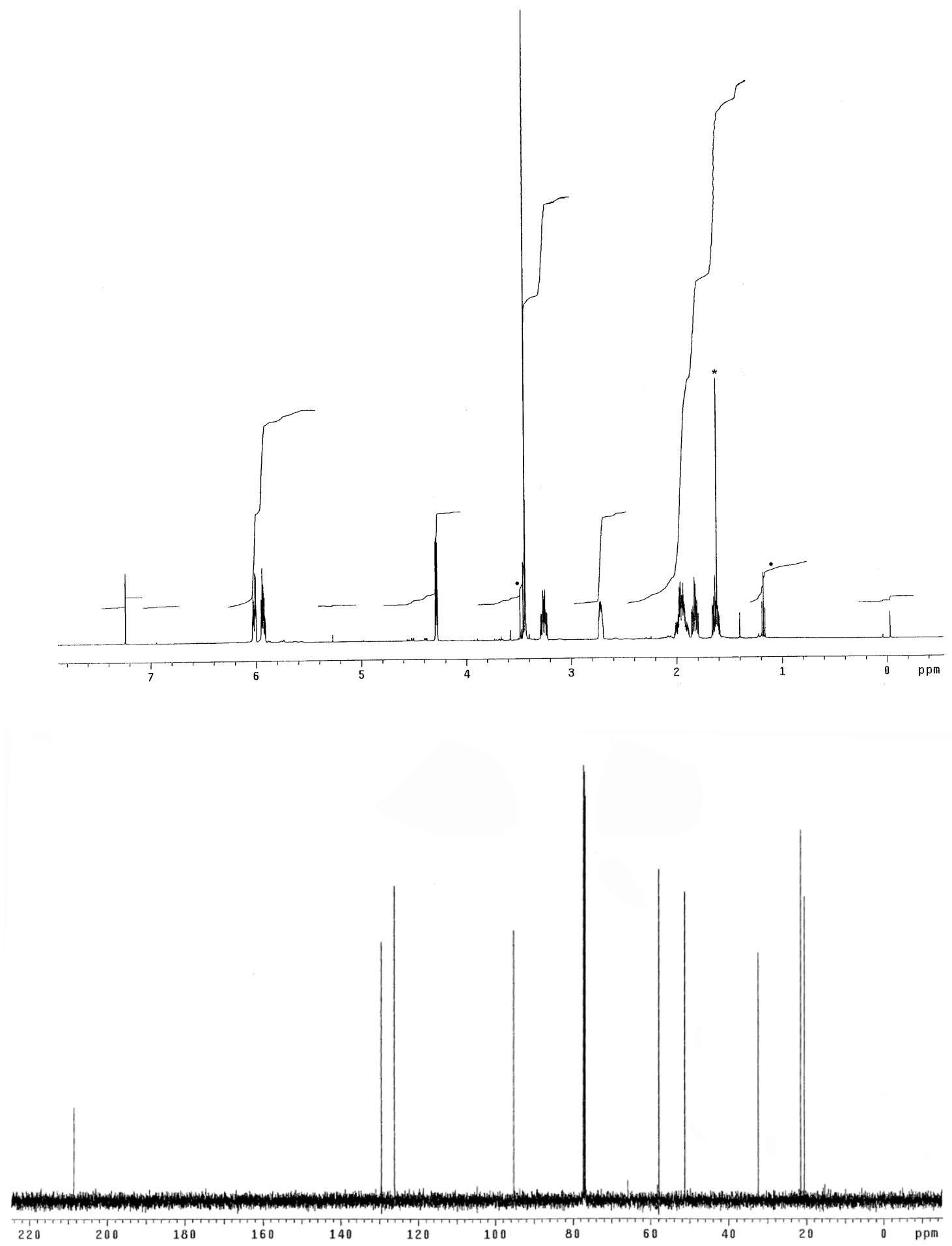

Figure S1. ${ }^{1} \mathrm{H}$ - and ${ }^{13} \mathrm{C}-\mathrm{NMR}$ Spectra of 8-exo-Methoxybicyclo[4.2.0]oct-2-en-7-one (3a) 


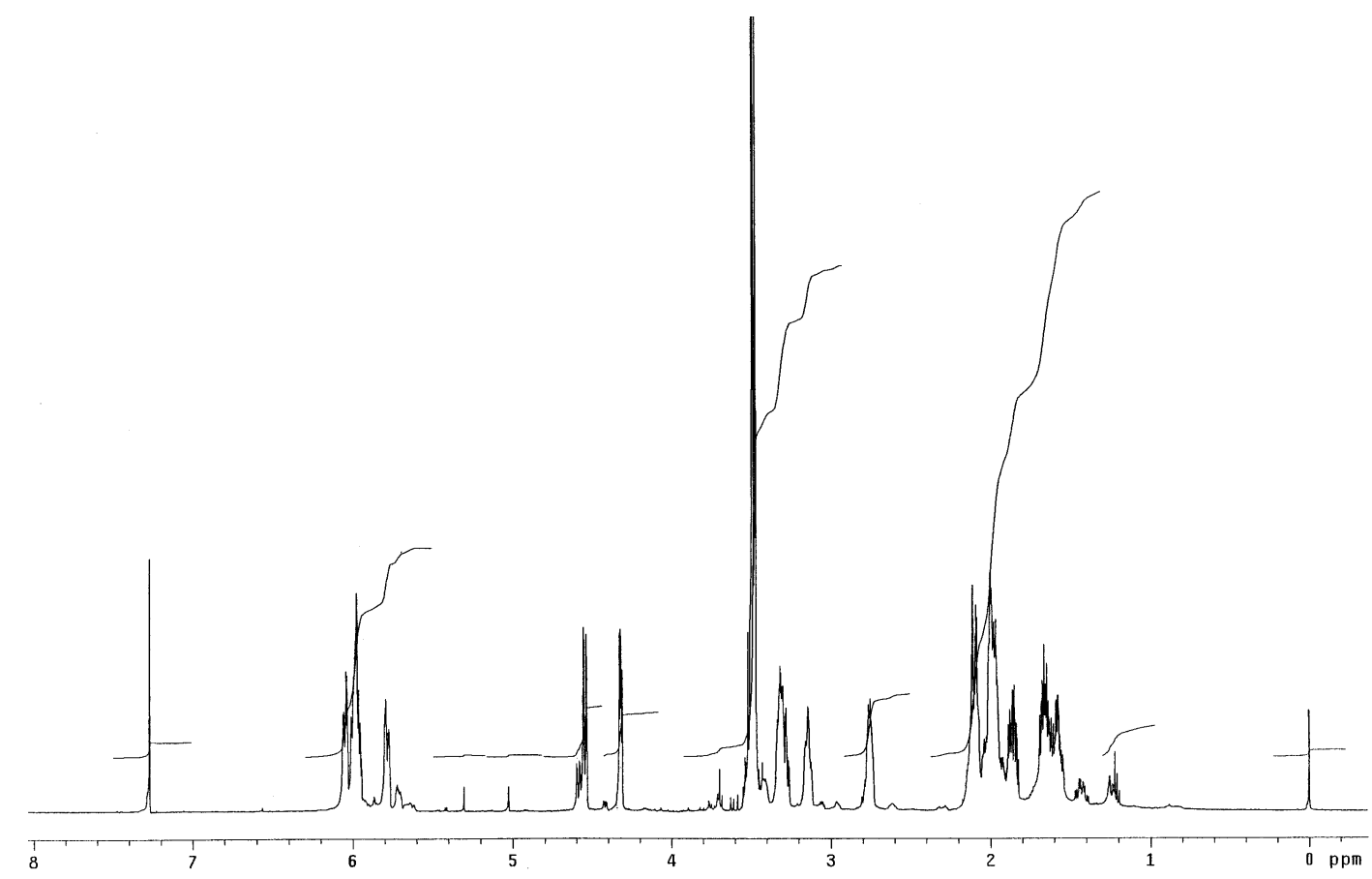

- impurity

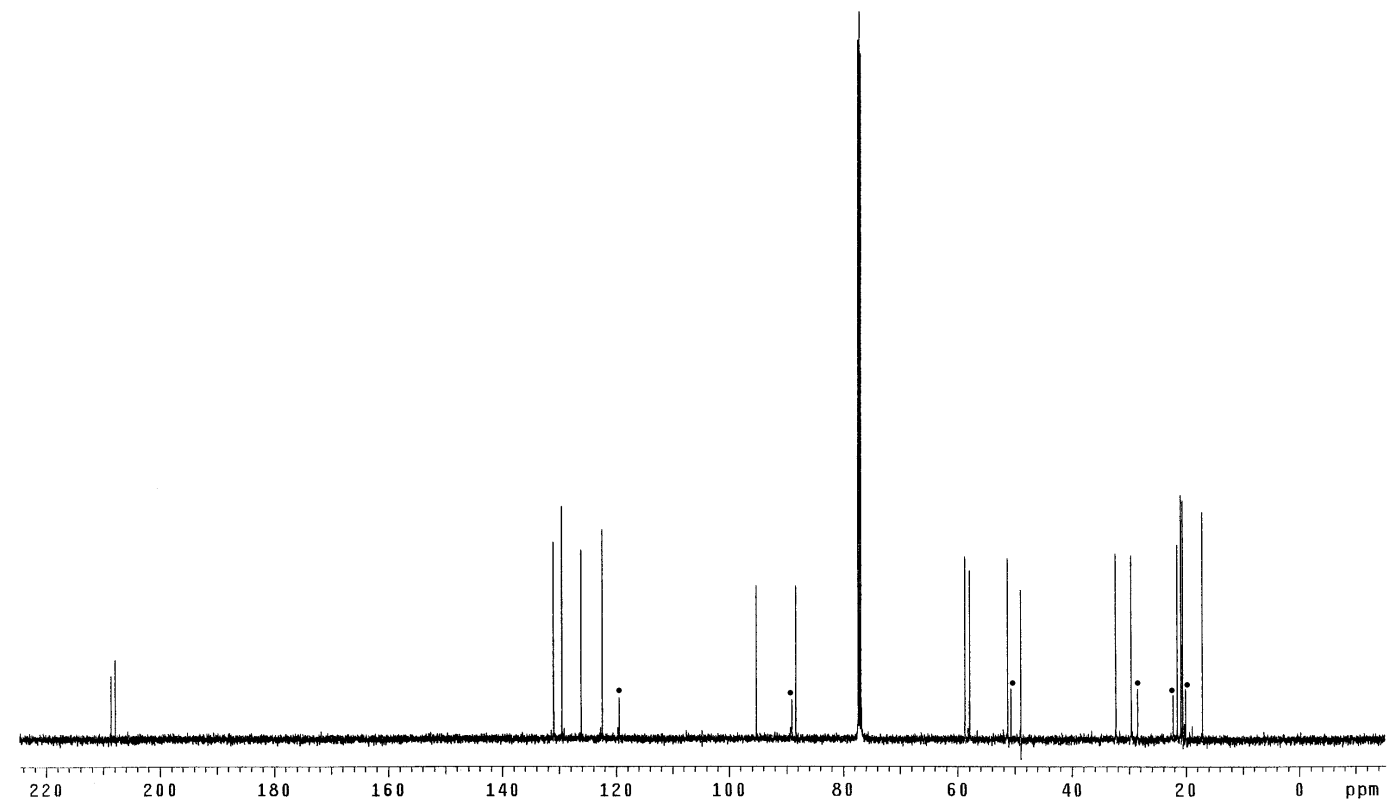

Figure S2. ${ }^{1} \mathrm{H}$ - and ${ }^{13} \mathrm{C}$-NMR Spectra of 8-endo-Methoxybicyclo[4.2.0]oct-2-en-7-one (3b) contaminated with 8-exo-Methoxybicyclo[4.2.0]oct-2-en-7-one (3a) 
- acetone
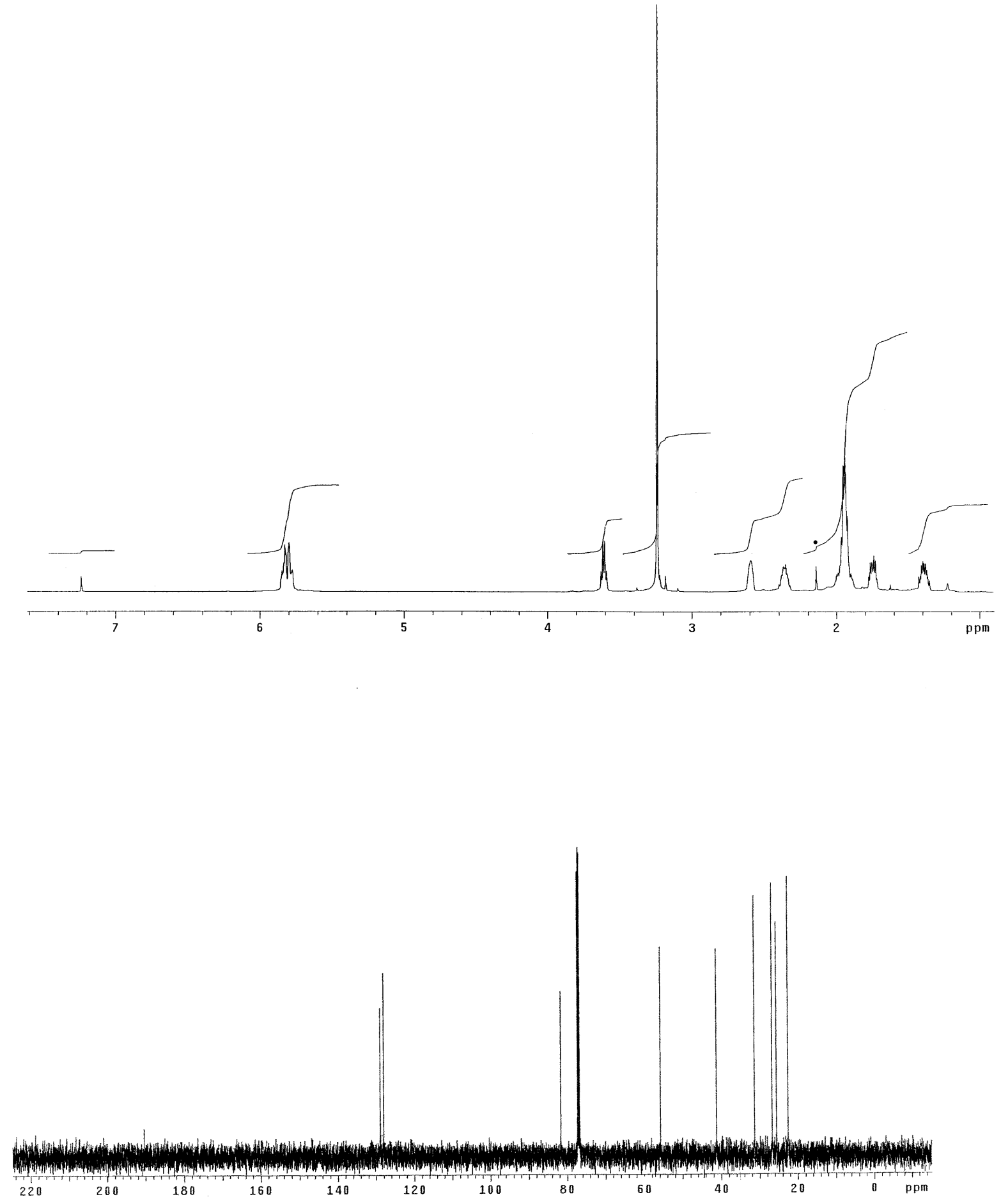

Figure S3. ${ }^{1} \mathrm{H}$ - and ${ }^{13} \mathrm{C}-\mathrm{NMR}$ Spectra of 8-exo-Methoxybicyclo[4.2.0]oct-2-ene (1a) 


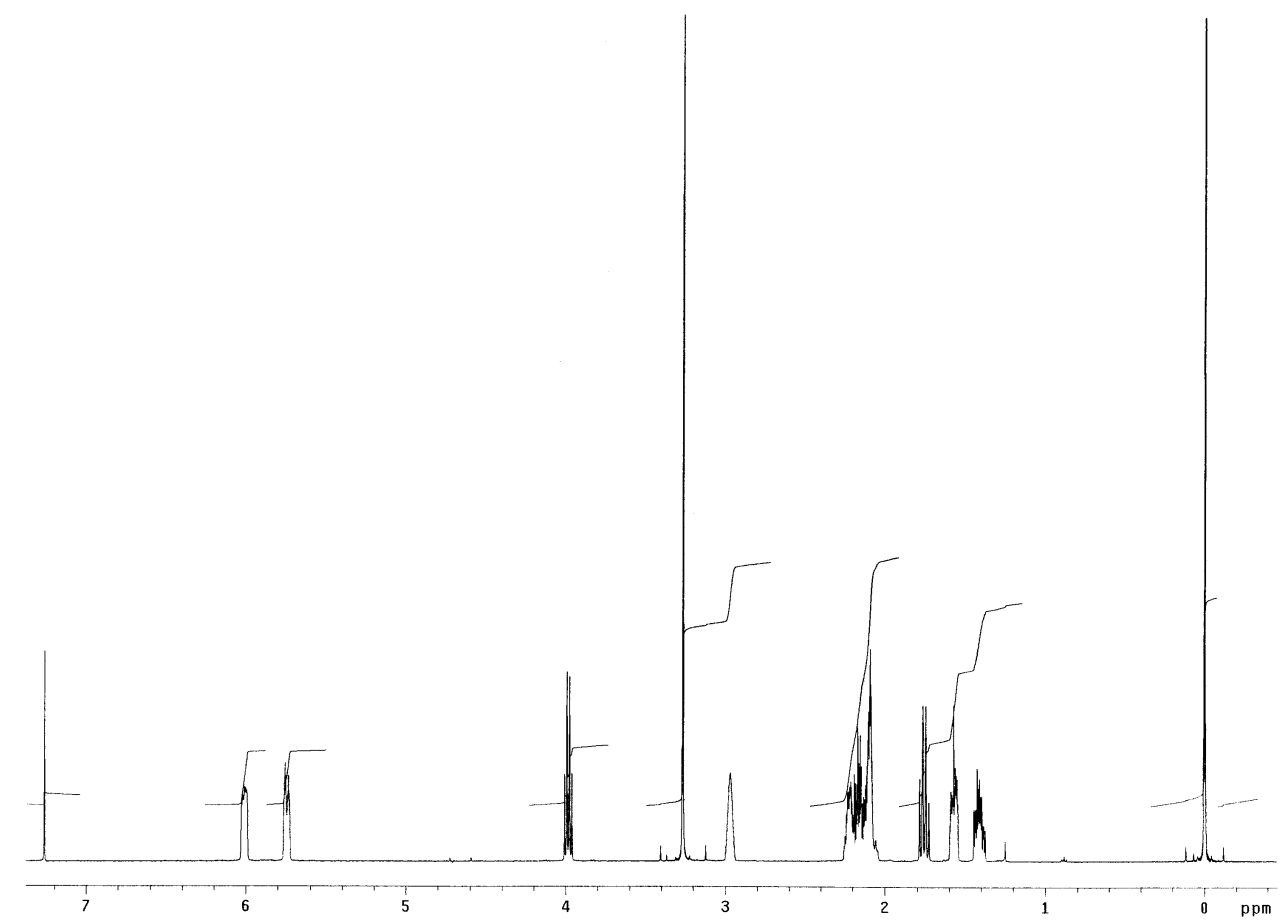

- 1b peak inside $\mathrm{CDCl}_{3}$ triplet

* two overlapping peaks

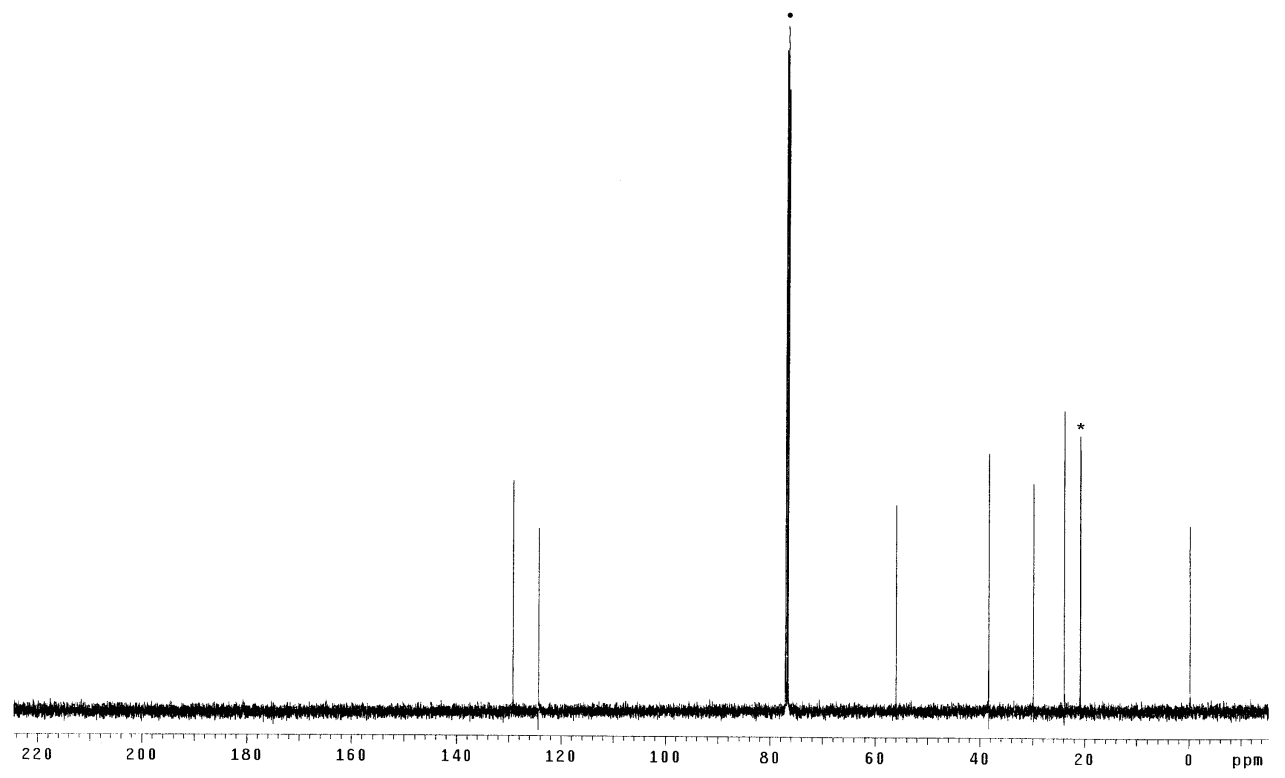

Figure S4. ${ }^{1} \mathrm{H}$ - and ${ }^{13} \mathrm{C}-\mathrm{NMR}$ Spectra of 8-endo-Methoxybicyclo[4.2.0]oct-2-ene (1b) 

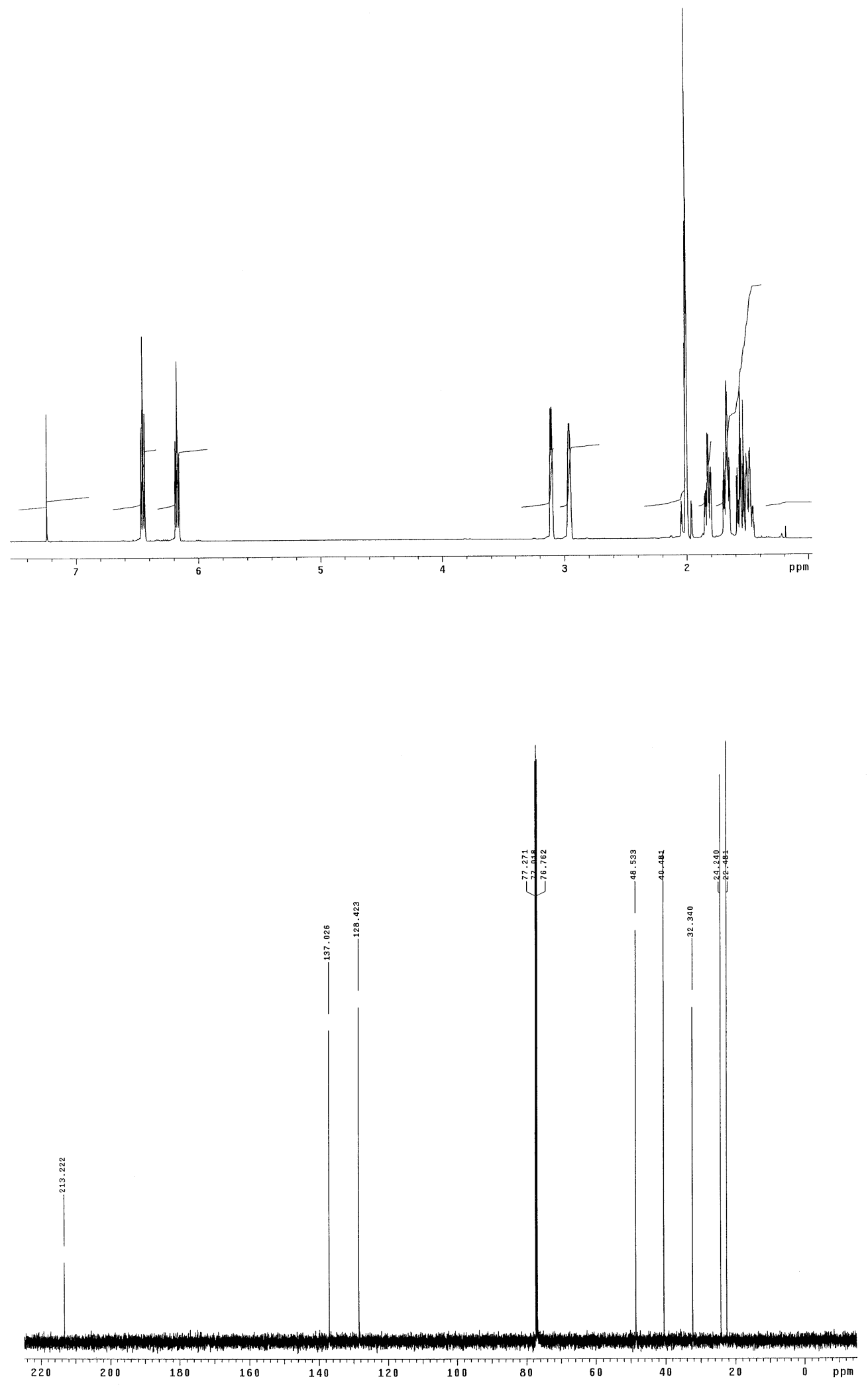

Figure S5. ${ }^{1} \mathrm{H}$ - and ${ }^{13} \mathrm{C}-\mathrm{NMR}$ Spectra of Bicyclo[2.2.2] oct-5-en-2-one (5) 

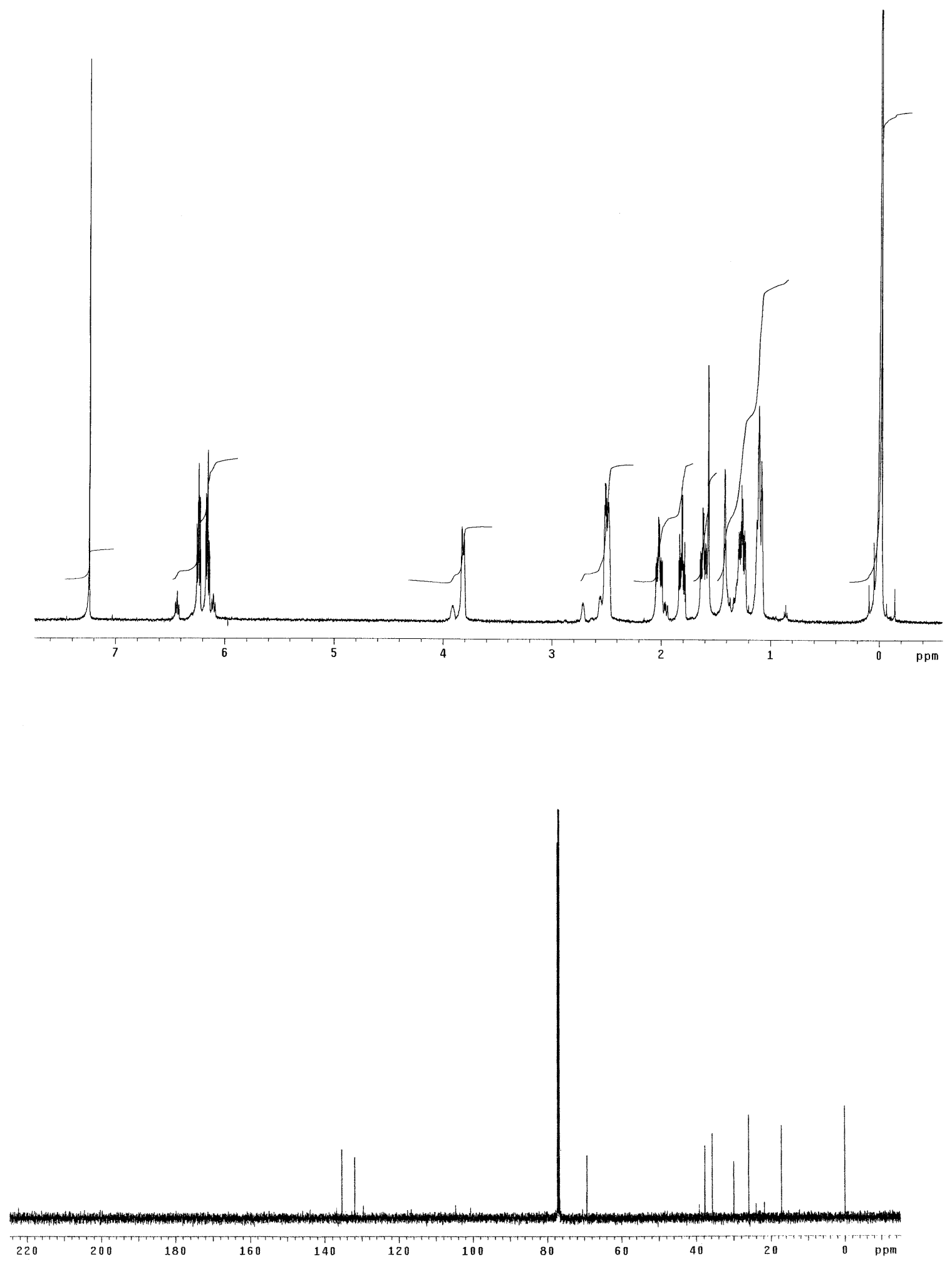

Figure S6. ${ }^{1} \mathrm{H}-$ and ${ }^{13} \mathrm{C}-\mathrm{NMR}$ Spectra of exo-Bicyclo[2.2.2]oct-5-en-2-ol (6a) 


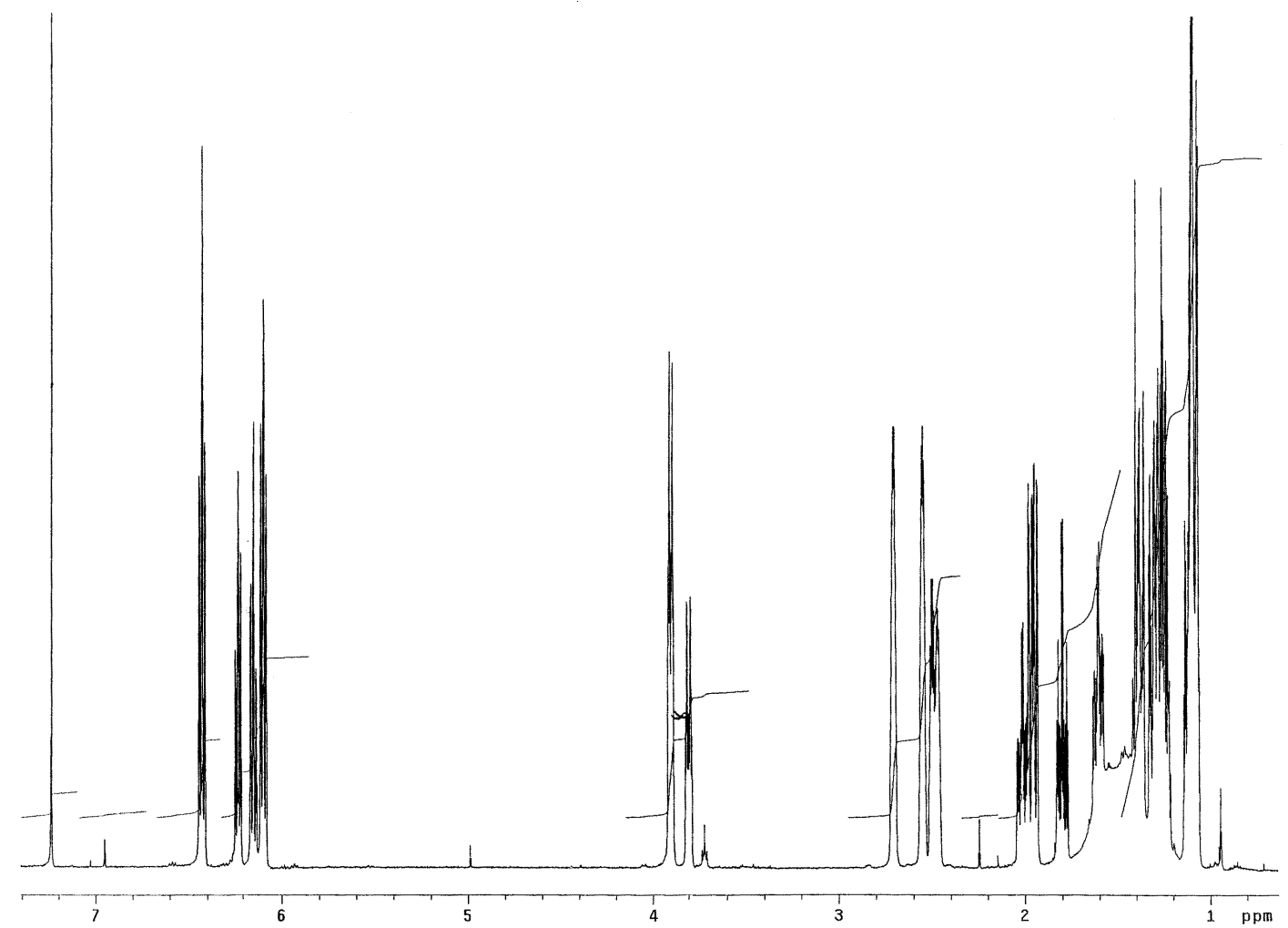

* two overlapping peaks $(\mathbf{6 a} / \mathbf{6 b})$

- peaks from 6a

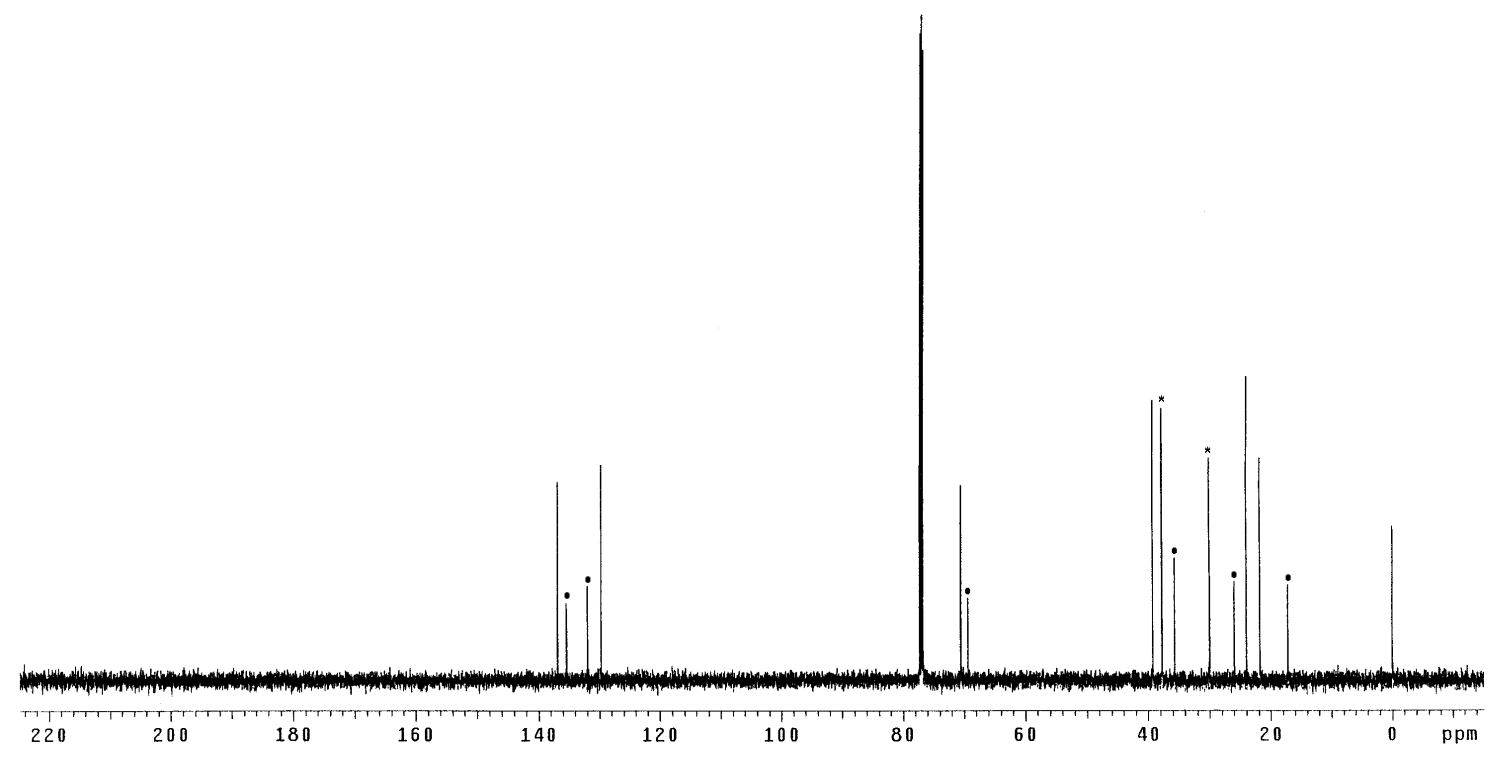

Figure S7. ${ }^{1} \mathrm{H}$ - and ${ }^{13} \mathrm{C}-\mathrm{NMR}$ Spectra of endo-Bicyclo[2.2.2] oct-5-en-2-ol (6b) contaminated with exo-Bicyclo[2.2.2]oct-5-en-2-ol (6a) 

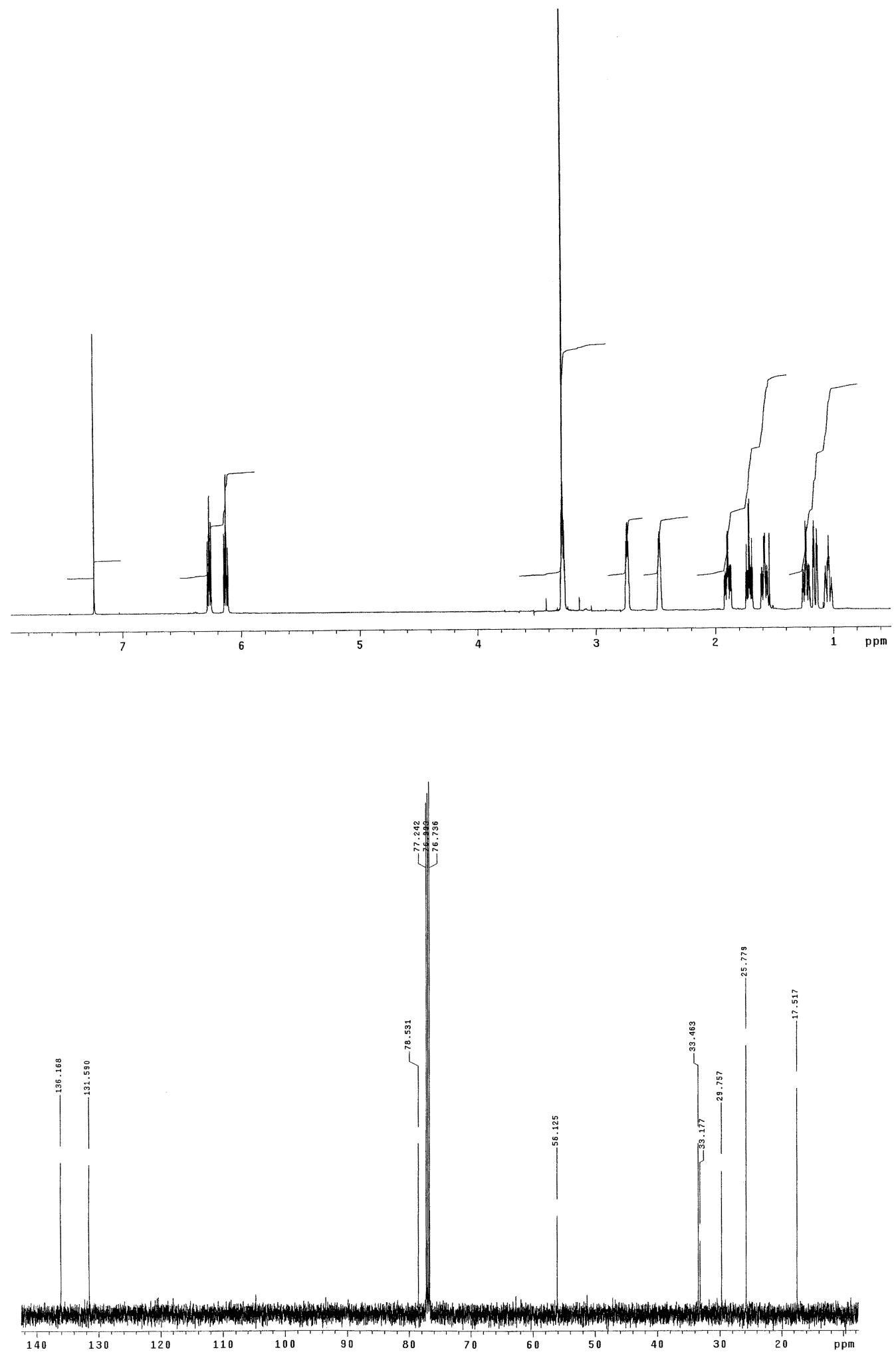

Figure S8. ${ }^{1} \mathrm{H}$ - and ${ }^{13} \mathrm{C}-\mathrm{NMR}$ Spectra of 5-exo-Methoxybicyclo[2.2.2] oct-2-ene (2a) 

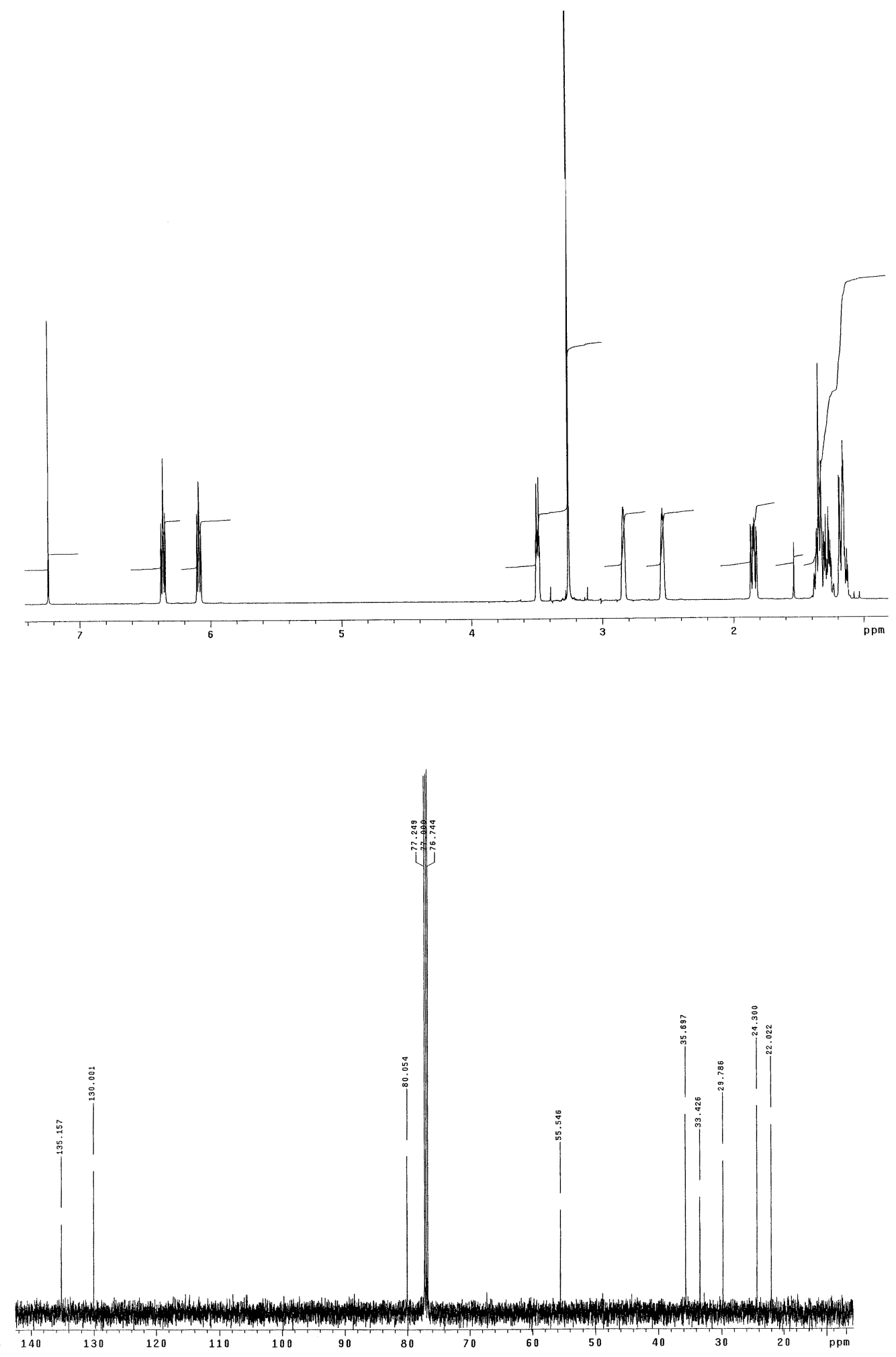

Figure S9. ${ }^{1} \mathrm{H}$ - and ${ }^{13} \mathrm{C}-\mathrm{NMR}$ Spectra of 5-endo-Methoxybicyclo[2.2.2] oct-2-ene (2b) 
- $\mathrm{Et}_{2} \mathrm{O}$

$* \mathrm{H}_{2} \mathrm{O}$

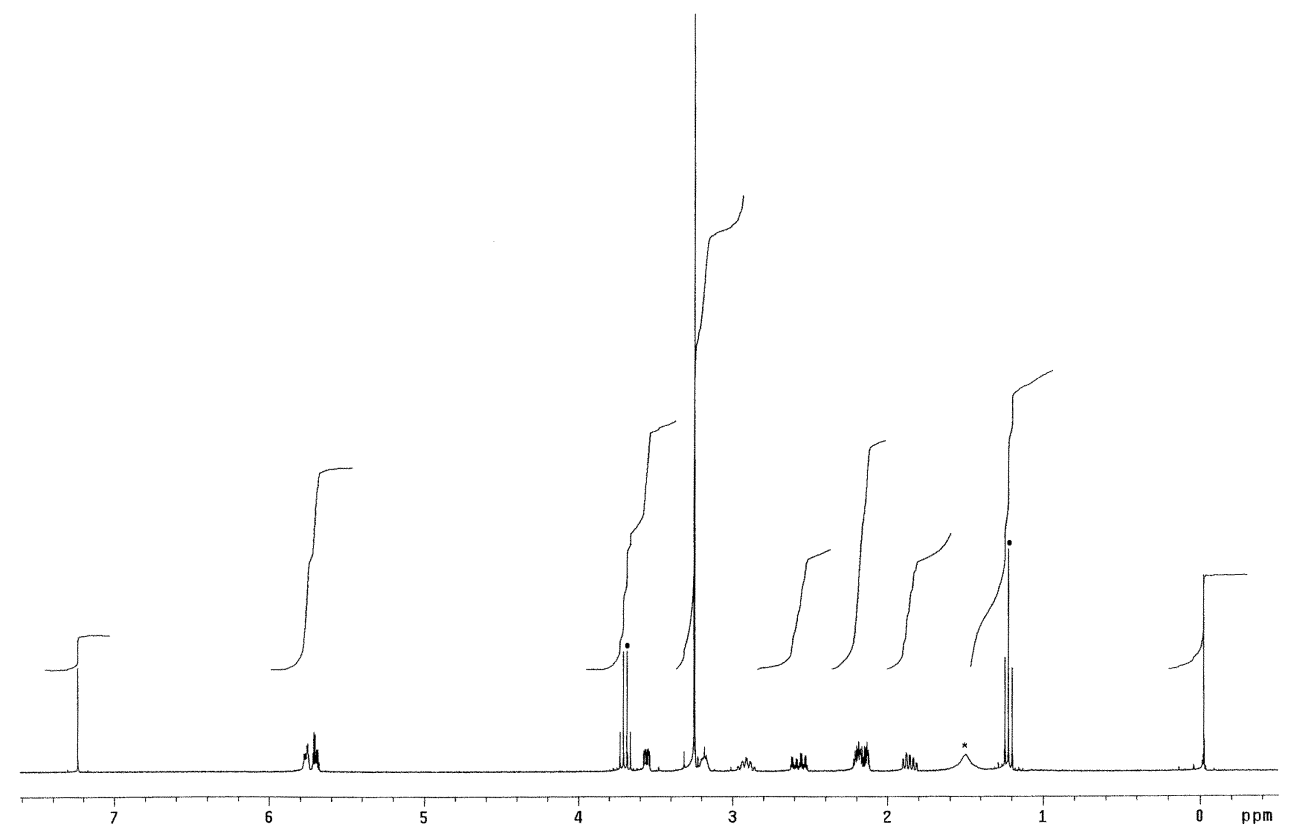

- electronic glitch

* acetone

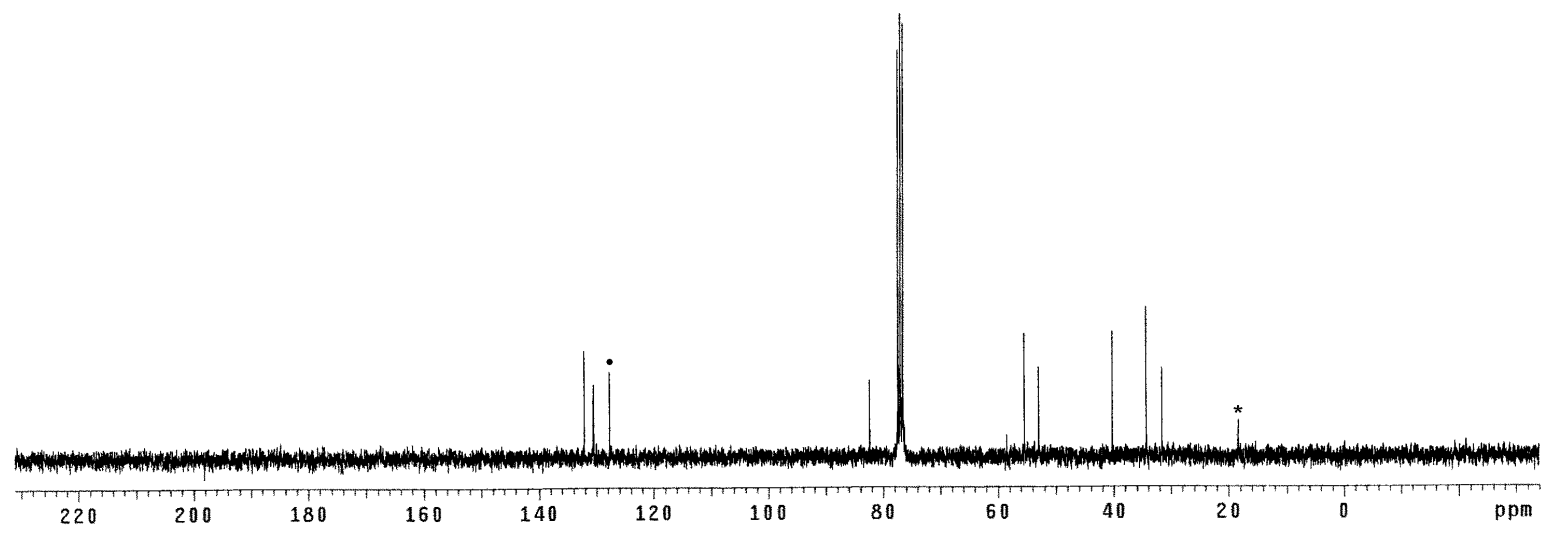

Figure S10. ${ }^{1} \mathrm{H}$ - and ${ }^{13} \mathrm{C}-\mathrm{NMR}$ Spectra of 7-exo-Methoxybicyclo[3.2.0]hept-2-ene (7a) 
- $\mathrm{Et}_{2} \mathrm{O}$
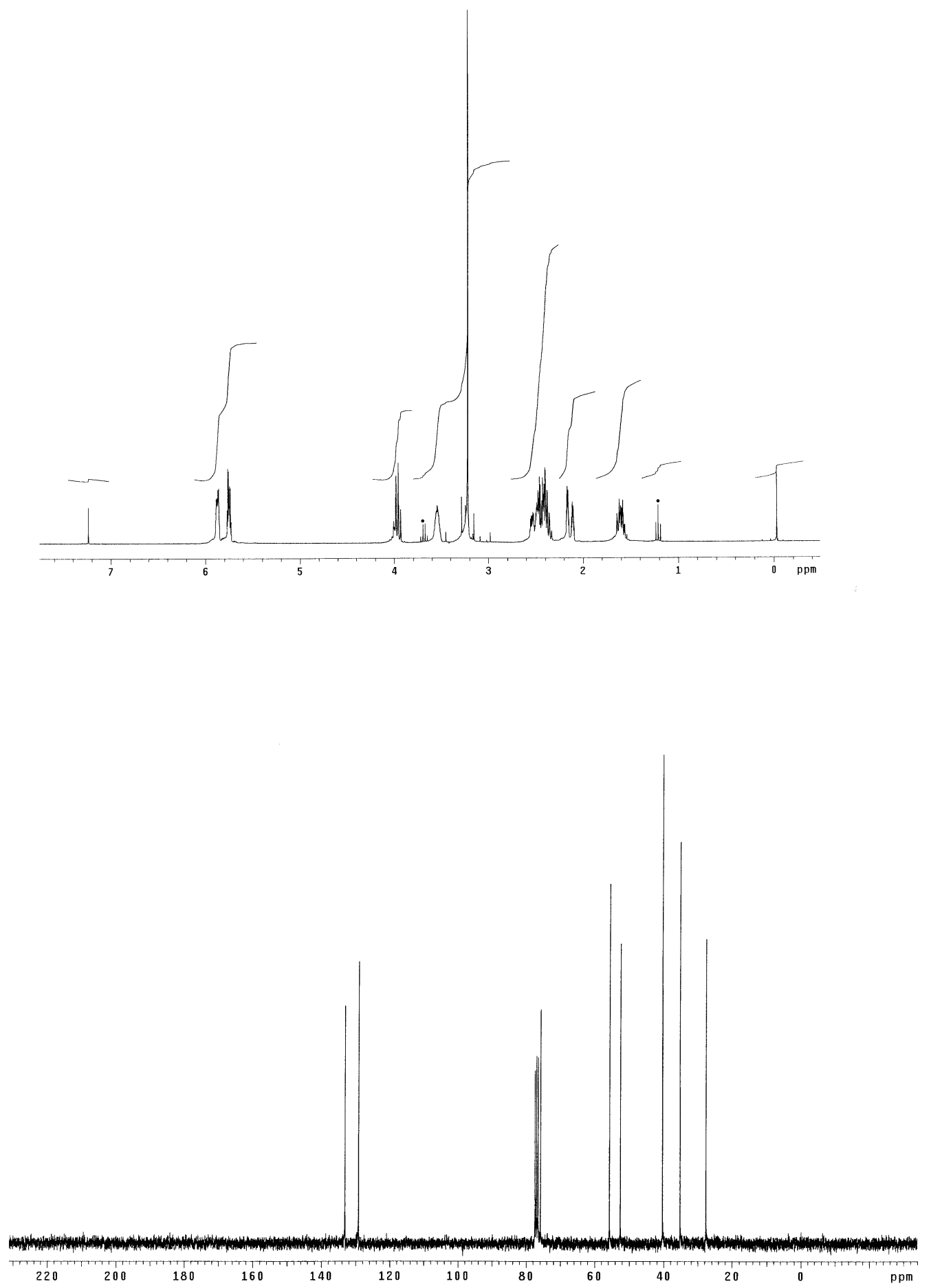

Figure S11. ${ }^{1} \mathrm{H}$ - and ${ }^{13} \mathrm{C}-\mathrm{NMR}$ Spectra of 7-endo-Methoxybicyclo[3.2.0]hept-2-ene (7b) 
- $\mathrm{Et}_{2} \mathrm{O}$
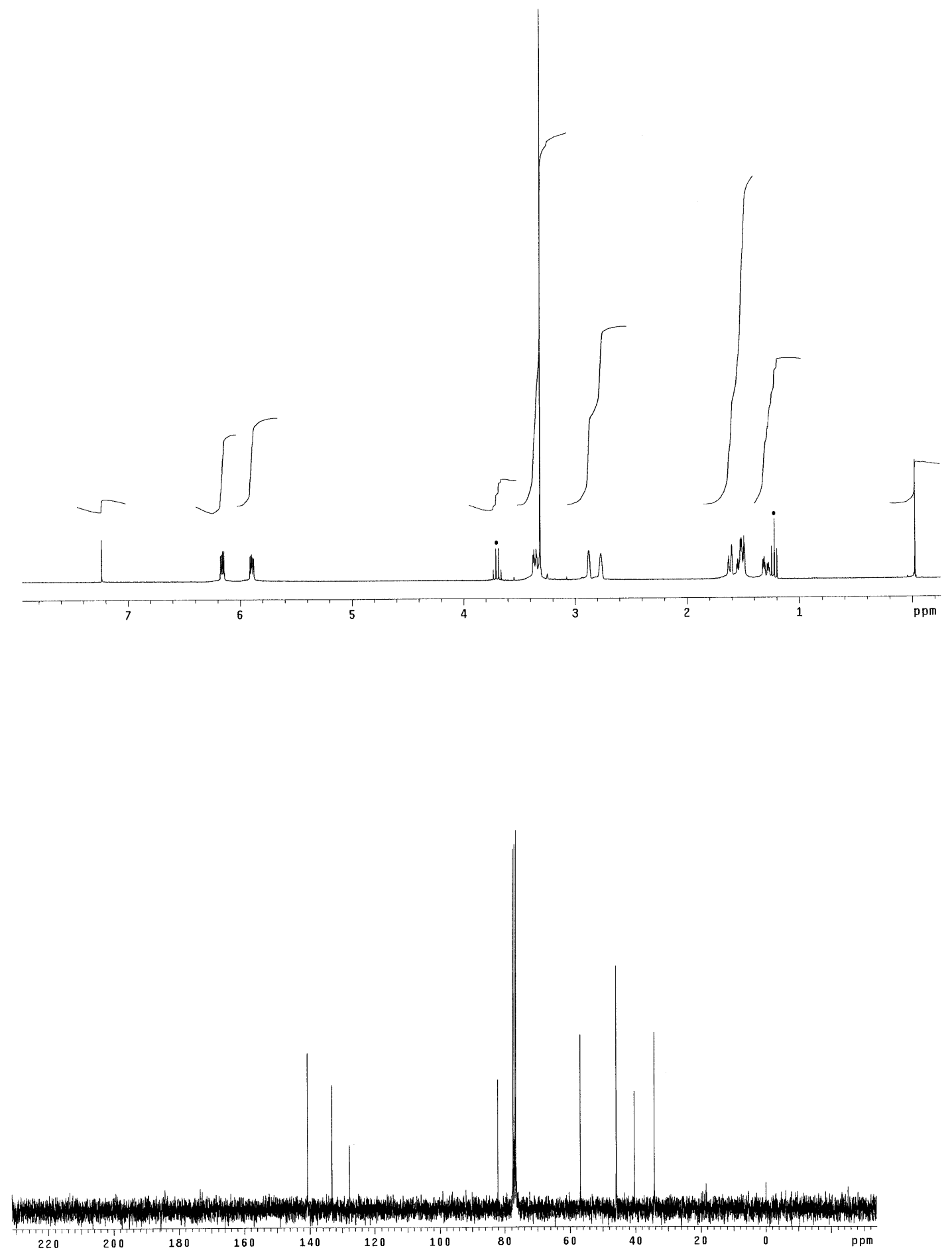

Figure S12. ${ }^{1} \mathrm{H}$ - and ${ }^{13} \mathrm{C}-\mathrm{NMR}$ Spectra of 5-exo-Methoxybicyclo[2.2.1]hept-2-ene (8a) 

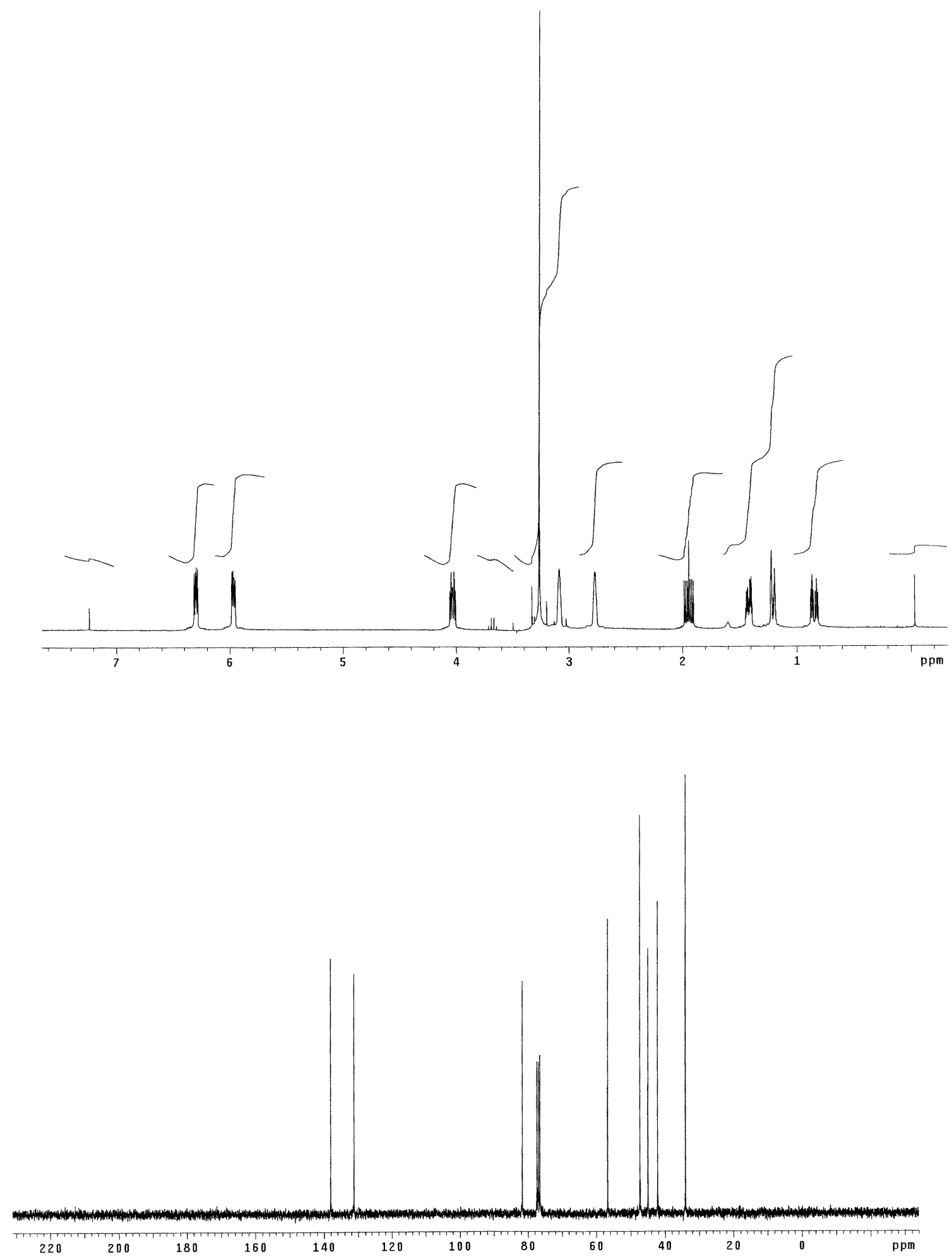

Figure S13. ${ }^{1} \mathrm{H}$ - and ${ }^{13} \mathrm{C}-\mathrm{NMR}$ Spectra of 5-endo-Methoxybicyclo[2.2.1]hept-2-ene (8b) 
Table S1. GC-Based Kinetic Data for Thermal Reaction of 1 a at $250{ }^{\circ} \mathrm{C}$ (concentrations expressed as mole fractions)

\begin{tabular}{|c|c|c|c|c|c|}
\hline time $(\mathrm{s})$ & $1 \mathrm{a}$ & $1 \mathrm{~b}$ & $2 \mathrm{a}$ & $2 \mathrm{~b}$ & 1,3 -cyclohexadiene \\
\hline \hline 0 & 1.000 & 0 & 0 & 0 & 0 \\
\hline $44,109.6$ & 0.808 & 0.107 & 0.045 & 0.014 & 0.027 \\
\hline $86,863.8$ & 0.626 & 0.170 & 0.080 & 0.028 & 0.096 \\
\hline $129,840.0$ & 0.539 & 0.150 & 0.110 & 0.031 & 0.171 \\
\hline
\end{tabular}

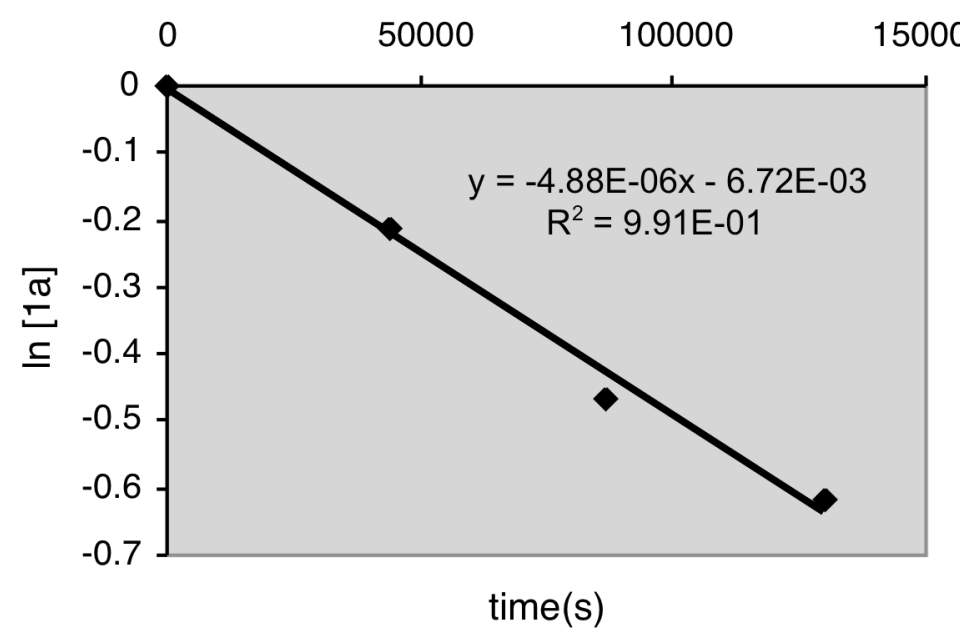

Figure S14. Kinetic Plot for Thermal Reaction of $19 @ 250{ }^{\circ} \mathrm{C}$ 
Table S2. GC-Based Kinetic Data for Thermal Reaction of $1 \mathrm{a}$ at $275^{\circ} \mathrm{C}$ (concentrations expressed as mole fractions)

\begin{tabular}{|c|c|c|c|c|c|}
\hline time $(\mathrm{s})$ & $1 \mathrm{a}$ & $1 \mathrm{~b}$ & $2 \mathrm{a}$ & $2 \mathrm{~b}$ & 1,3-Cyclohexadiene \\
\hline \hline 0 & 1.000 & 0 & 0 & 0 & 0 \\
\hline $7,224.6$ & 0.767 & 0.105 & 0.053 & 0.018 & 0.057 \\
\hline $14,413.8$ & 0.573 & 0.142 & 0.102 & 0.030 & 0.154 \\
\hline $28,812.0$ & 0.366 & 0.123 & 0.158 & 0.048 & 0.305 \\
\hline $43,216.8^{a}$ & 0.326 & 0.089 & 0.199 & 0.059 & 0.328 \\
\hline $59,104.8^{a}$ & 0.271 & 0.054 & 0.204 & 0.060 & 0.412 \\
\hline
\end{tabular}

$a$ Last two kinetic points excluded in Figure S15 due to epimerization of $\mathbf{1 b}$ back to $\mathbf{1 a}$.

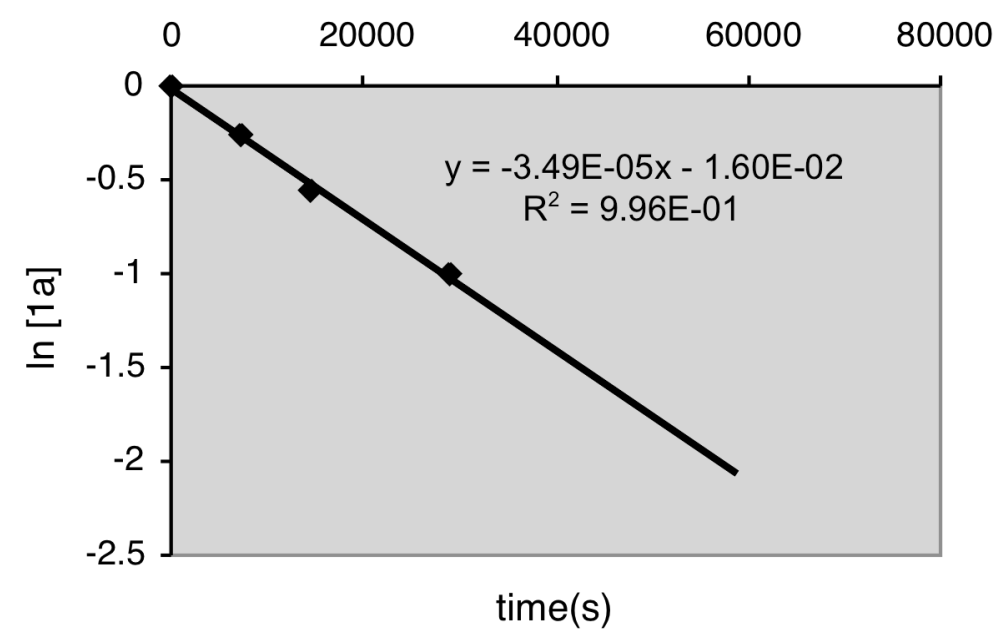

Figure S15. Kinetic Plot for Thermal Reaction of $1 \mathbf{a} @ 275^{\circ} \mathrm{C}$ 
Table S3. GC-Based Kinetic Data for Thermal Reaction of 1 a at $300{ }^{\circ} \mathrm{C}$ (concentrations expressed as mole fractions)

\begin{tabular}{|c|c|c|c|c|c|}
\hline time $(\mathrm{s})$ & $1 \mathrm{a}$ & $1 \mathrm{~b}$ & $2 \mathrm{a}$ & $2 \mathrm{~b}$ & 1,3 -cyclohexadiene \\
\hline 0 & 1.000 & 0 & 0 & 0 & 0 \\
\hline $3,606.0$ & 0.479 & 0.115 & 0.149 & 0.051 & 0.202 \\
\hline $7,209.0$ & 0.269 & 0.062 & 0.244 & 0.083 & 0.340 \\
\hline $11,033.4$ & 0.143 & 0.016 & 0.278 & 0.092 & 0.472 \\
\hline
\end{tabular}

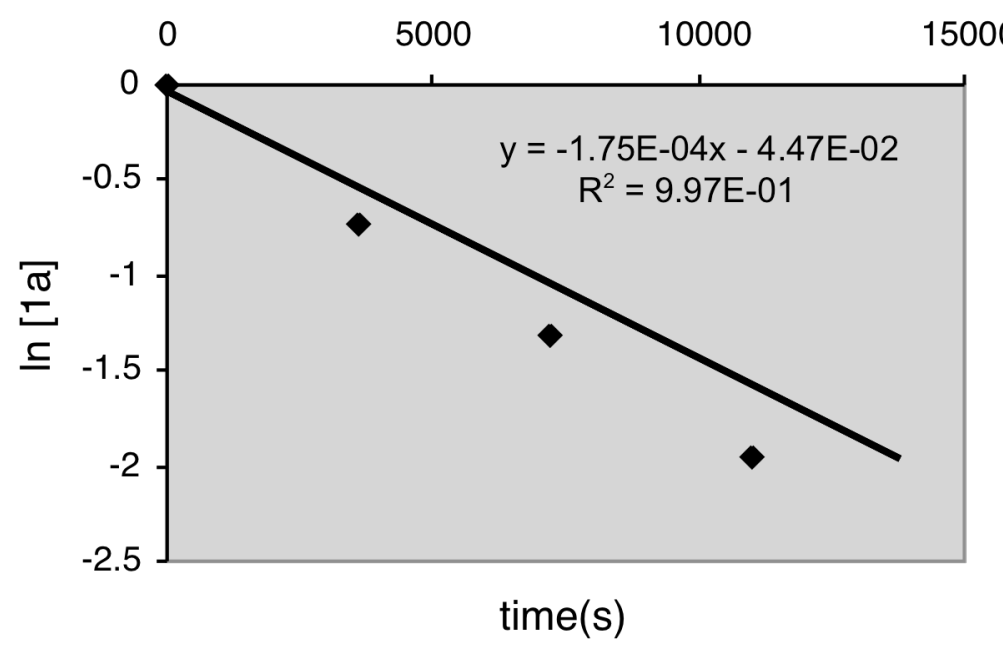

Figure S16. Kinetic Plot for Thermal Reaction of 1a @ $300{ }^{\circ} \mathrm{C}$ 
Table S4. GC-Based Kinetic Data for Thermal Reaction of $1 \mathrm{~b}$ at $250{ }^{\circ} \mathrm{C}$ (concentrations expressed as mole fractions)

\begin{tabular}{|c|c|c|c|c|c|}
\hline time $(\mathrm{s})$ & $1 \mathrm{a}$ & $1 \mathrm{~b}$ & $2 \mathrm{a}$ & $2 \mathrm{~b}$ & 1,3-cyclohexadiene \\
\hline \hline 0 & 0 & 1.000 & 0 & 0 & 0 \\
\hline $43,535.4$ & 0.205 & 0.648 & 0.032 & 0.004 & 0.111 \\
\hline $86,416.8$ & 0.281 & 0.426 & 0.060 & 0.009 & 0.224 \\
\hline $129,845.4$ & 0.268 & 0.303 & 0.079 & 0.015 & 0.336 \\
\hline
\end{tabular}

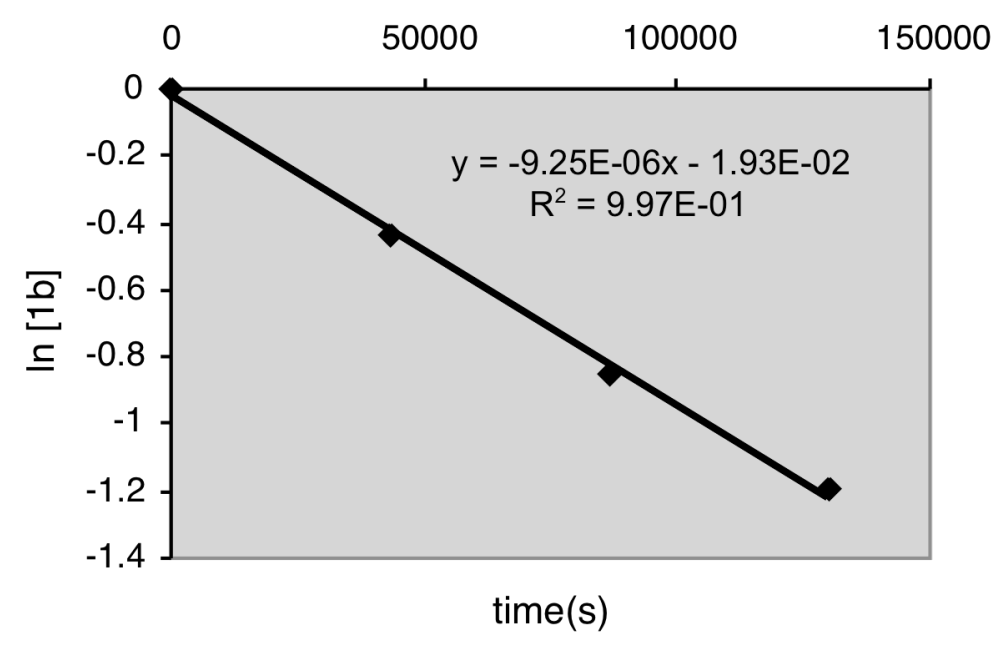

Figure S17. Kinetic Plot for Thermal Reaction of $\mathbf{1 b} @ 250{ }^{\circ} \mathrm{C}$ 
Table S5. GC-Based Kinetic Data for Thermal Reaction of $1 \mathrm{~b}$ at $275^{\circ} \mathrm{C}$ (concentrations expressed as mole fractions)

\begin{tabular}{|c|c|c|c|c|c|}
\hline time $(\mathrm{s})$ & $1 \mathrm{a}$ & $1 \mathrm{~b}$ & $2 \mathrm{a}$ & $2 \mathrm{~b}$ & 1,3 -cyclohexadiene \\
\hline \hline 0 & 0 & 1.000 & 0 & 0 & 0 \\
\hline $1,808.4$ & 0.087 & 0.876 & trace & 0 & 0.029 \\
\hline $3,583.8$ & 0.148 & 0.740 & trace & 0 & 0.098 \\
\hline $7,177.8$ & 0.233 & 0.581 & 0.043 & 0 & 0.143 \\
\hline $10,917.0$ & 0.244 & 0.431 & 0.048 & trace & 0.264 \\
\hline $14,498.4$ & 0.301 & 0.347 & 0.084 & trace & 0.253 \\
\hline
\end{tabular}

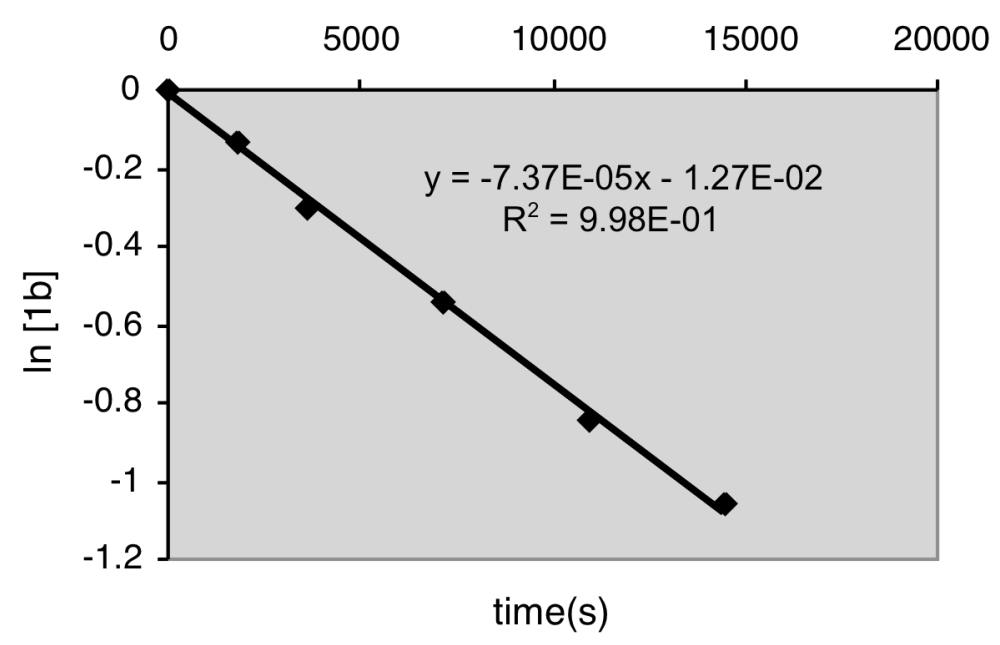

Figure S18. Kinetic Plot for Thermal Reaction of $\mathbf{1 b} @ 275^{\circ} \mathrm{C}$ 
Table S6. GC-Based Kinetic Data for Thermal Reaction of $1 \mathrm{~b}$ at $300^{\circ} \mathrm{C}$ (concentrations expressed as mole fractions)

\begin{tabular}{|c|c|c|c|c|c|}
\hline time $(\mathrm{s})$ & $1 \mathrm{a}$ & $1 \mathrm{~b}$ & $2 \mathrm{a}$ & $2 \mathrm{~b}$ & 1,3 -cyclohexadiene \\
\hline \hline 0 & 0 & 1.000 & 0 & 0 & 0 \\
\hline $1.810 \times 10^{3}$ & 0.227 & 0.433 & 0.058 & 0.014 & 0.268 \\
\hline $3.592 \times 10^{3}$ & 0.257 & 0.214 & 0.113 & 0.030 & 0.385 \\
\hline $5.445 \times 10^{3}$ & 0.186 & 0.183 & 0.127 & 0.032 & 0.472 \\
\hline $7.214 \times 10^{3}$ & 0.150 & 0.073 & 0.158 & 0.039 & 0.580 \\
\hline
\end{tabular}

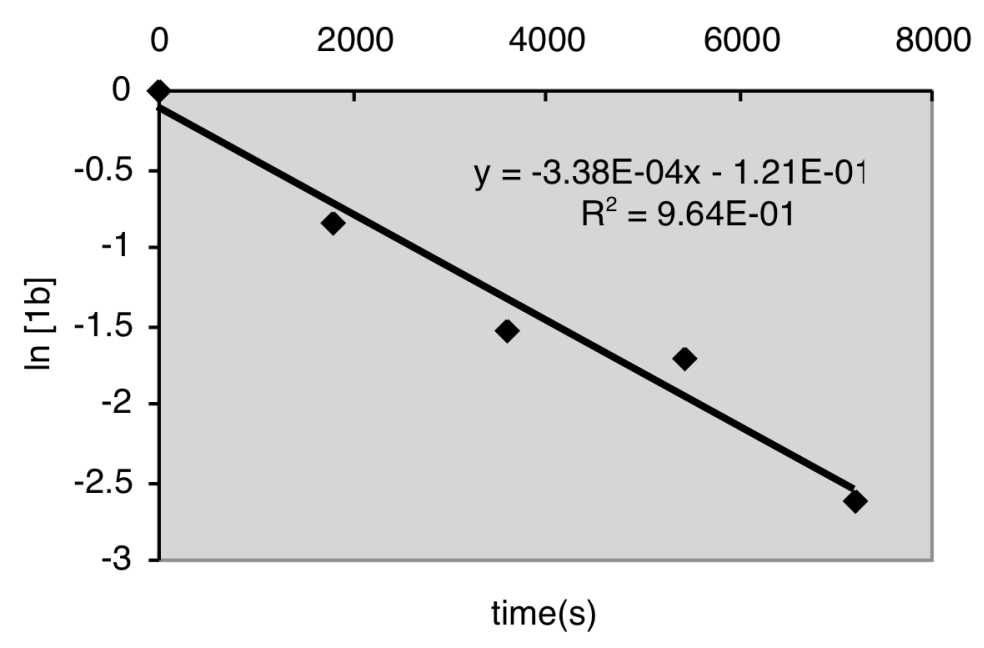

Figure S19. Kinetic Plot for Thermal Reaction of $1 \mathbf{b} @ 300{ }^{\circ} \mathrm{C}$ 
Table S7. Temperature-dependent Data for Thermal Reaction of 1a

\begin{tabular}{|c|c|c|c|c|}
\hline temp $\left({ }^{\circ} \mathrm{C}\right)$ & $\mathrm{T}(\mathrm{K})$ & $1 / \mathrm{T}\left(\mathrm{K}^{-1}\right)$ & $\mathrm{k}_{\mathrm{d}}\left(\mathrm{s}^{-1}\right)$ & $\log \mathrm{k}_{\mathrm{d}}$ \\
\hline \hline 250 & 523 & $1.912 \times 10^{-3}$ & $4.9 \times 10^{-6}$ & -5.310 \\
\hline 275 & 548 & $1.825 \times 10^{-3}$ & $3.5 \times 10^{-5}$ & -4.456 \\
\hline 300 & 573 & $1.745 \times 10^{-3}$ & $1.75 \times 10^{-4}$ & -3.757 \\
\hline
\end{tabular}

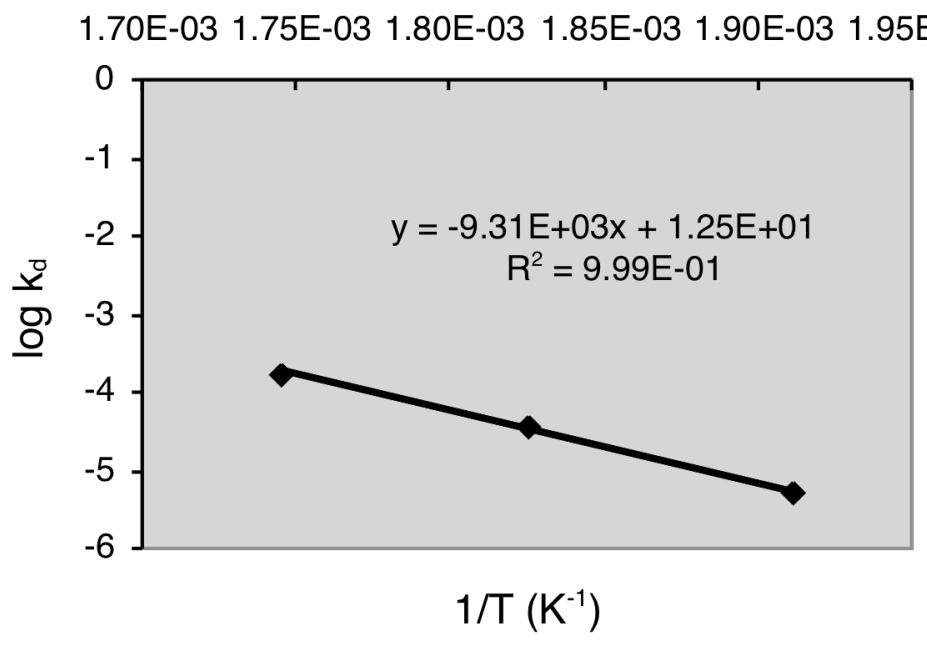

Figure S20. Arrhenius Plot for Thermal Reaction of 1a 
Table S8. Temperature-dependent Data for Thermal Reaction of $\mathbf{1 b}$

\begin{tabular}{|c|c|c|c|c|}
\hline temp $\left({ }^{\circ} \mathrm{C}\right)$ & $\mathrm{T}(\mathrm{K})$ & $1 / \mathrm{T}\left(\mathrm{K}^{-1}\right)$ & $\mathrm{k}_{\mathrm{d}}^{\prime}\left(\mathrm{s}^{-1}\right)$ & $\log \mathrm{k}_{\mathrm{d}}^{\prime}$ \\
\hline \hline 250 & 523 & $1.912 \times 10^{-3}$ & $9.3 \times 10^{-6}$ & -5.032 \\
\hline 275 & 548 & $1.825 \times 10^{-3}$ & $7.4 \times 10^{-5}$ & -4.131 \\
\hline 300 & 573 & $1.745 \times 10^{-3}$ & $3.4 \times 10^{-4}$ & -3.469 \\
\hline
\end{tabular}

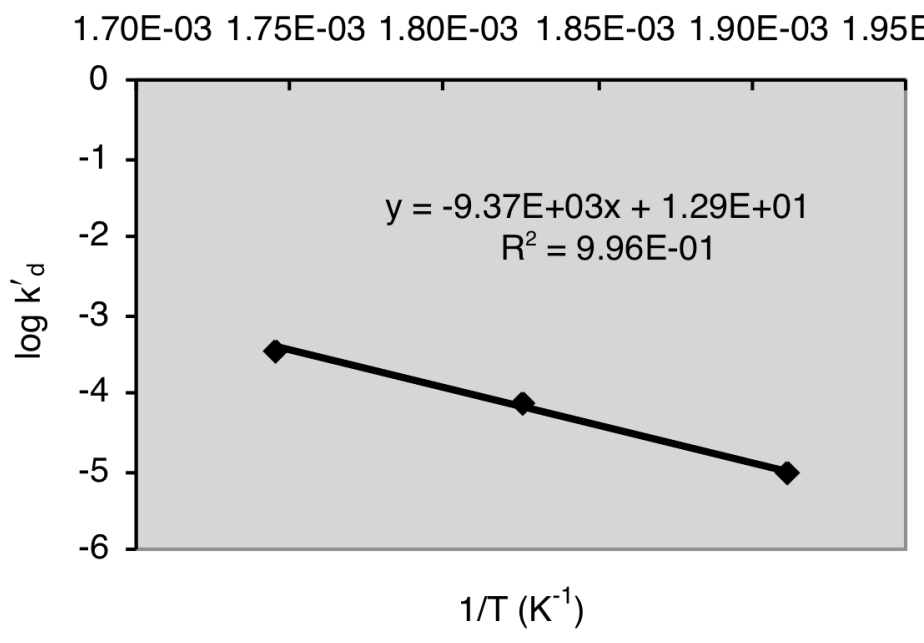

Figure S21. Arrhenius Plot for Thermal Reaction of $\mathbf{1 b}$ 


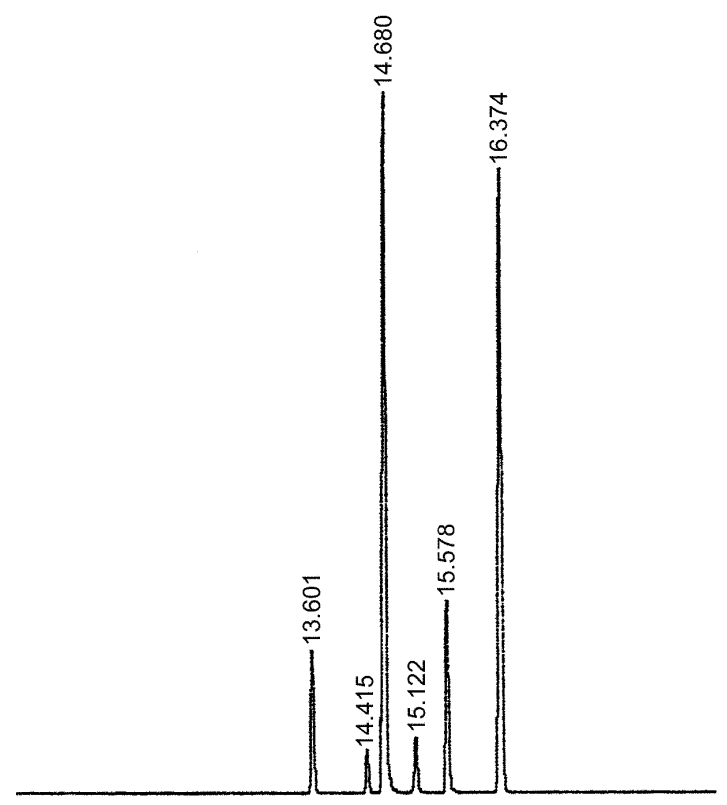

Figure S22. Capillary GC Chromatogram for Thermal Reaction of 1a @ $275{ }^{\circ} \mathrm{C}(240.23$ min) with Elution Order 2a, 2b, 1a, impurity (7-methoxybicyclo[4.2.0]octane), 1b, and internal standard (undecane).

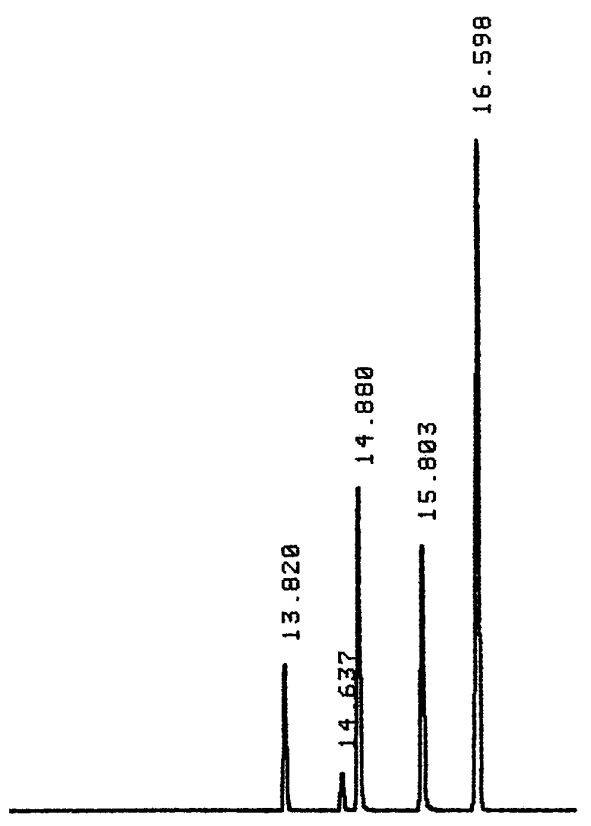

Figure S23. Capillary GC Chromatogram for Thermal Reaction of $\mathbf{1 b} @ 300{ }^{\circ} \mathrm{C}$ (59.86 min) with Elution Order 2a, 2b, 1a, 1b, and internal standard (undecane). 